\title{
3D Atom Probe for Nano-scale Chemical Analysis
}

\author{
A.N. Davis*, F. Horréard**, I. Martin**, R. Benbalagh**, L. Renaud ** \\ * Cameca Instruments Inc., 91 McKee Drive, Mahwah, NJ 07430, USA \\ ** Cameca, 29 quai des Grésillons, 92622 Gennevilliers Cedex, France
}

The aim of this presentation is to review today's applications of 3-Dimensional Atom Probe Tomography (or, 'APT') including metals, GST Phase Change Materials, ultra-thin, multi-layer structures of semiconductors and insulators, and ceramics/refractory crystals. Access to analytical success has become possible with recent instrumentation developments such as the use of high frequency femtosecond UV laser pulsing (in addition to other wavelengths).

Potentials and limits of APT will be emphasized. These include important instrumental requirements associated with the physical field evaporation process: resolving multiple detector hits (several atoms evaporated during the same pulse) thus requiring a fast detection system, reduction of thermal effects that degrade mass resolution, improvement of instrumental transmission (cluster composition measurements, e.g.), and further technical advancements.

APT can be considered as the only technique which offers capabilities for both 3D imaging and chemical composition measurements at the atomic scale. The sample is prepared as a tip of few tens of $\mathrm{nm}$ end radius. It is introduced in a UHV chamber, cooled down to $20 \mathrm{~K}$ to minimize thermal agitation of the atoms, and polarized at a few $\mathrm{kV}$. This induces a huge electrostatic field (few tens of $\mathrm{V} / \mathrm{nm}$ ) at the apex. Pulsing the voltage above a threshold will field-evaporate atoms at precise times. Atoms will then be ionized in the E-field and accelerated toward a Position Sensitive Detector.

A 3D-AP is essentially a projection microscope coupled with a time-of-flight mass spectrometer. Indeed, measuring the time-of-flight between a voltage pulse and the arrival at the detector will give a mass-over-charge ratio, and measuring an ion's impact on the detector will determine the original position of the atom on the tip surface. It is then possible to reconstruct a quantitative chemical 3D image of atoms at the tip, with sub-nm spatial resolution $(0.1-0.2 \mathrm{~nm}$ in depth and $0.3-0.5 \mathrm{~nm}$ laterally). Since its early development, 3D Atom Probe has provided major contributions in materials science [1]. But up until the last few years, APT studies were limited to conductive materials (HV pulses cannot be efficiently propagated along a tip of non-conductive materials). Furthermore, 'traditional' sample preparation methods made it difficult to prepare an APT tip from a specific area of interest in a bulk material.

By now, these drawbacks have been largely overcome and the field of investigation of this technique has been extended. The major breakthrough has been the introduction of LASER pulsing mode in addition to traditional HV-pulsed evaporation mode. Materials of low conductivity is now possible, thanks to laser pulsing. Moreover, with the help of Focused Ion Beam (FIB) techniques, it is now possible to extract and prepare a sample ready for 3D-AP analysis from a selected site-specific location of a bulk sample or from a piece of wafer.

Reference

[1] D. Blavette et al., Science, 286, (1999) 5448. 